scale surfaces. Observed mechanical properties correlated with the known composition of each layer. The researchers concluded that layer thickness, grading of mechanical properties within and between layers, as well as material layer sequence within individual scales of $P$. senegalus are physiologically important for abating penetration and facilitating protection while yielding biomechanical flexibility and maneuverability of the entire fish. Materials design principles learned from these "living fossils" may hold promise for improved design of engineered biomimetic structural materials, such as protective armor for humans or military vehicle applications, said the researchers.

SAMESHA R. BARNES

\section{Spatial Light Scattering Reveals New Information on Atmospheric Ice Crystals}

Uncertainty about the future of Earth's climate has opened avenues of research into the elements shaping the climate. Meteorological evidence suggests that there are correlations between cloud properties and climate change. Clouds, comprised mainly of water vapor, droplets, and ice crystals, both absorb heat and reflect radiation from the sun. However, the magnitude of their ability to alter climate is unknown. What is known is that the size, shape, and number of particles present in the cloud determines its radiative properties. Currently, analysis of these features is frequently performed by instruments such as the Cloud Particle Imager (CPI), which uses a pulsing laser to capture real images of ice crystals on a CCD camera. However, the CPI is limited to imaging particles greater than $\sim 25 \mu \mathrm{m}$. The use of the device described in this study will allow data on particle size, shape, and quantity to be acquired from particles approaching $1 \mu \mathrm{m}$.

P.H. Kaye and colleagues at the University of Hertfordshire, P.J. DeMott of Colorado State University, and C. Saunders and colleagues at the University of Manchester have used a light scattering approach to analyze atmospheric ice crystals. In the July 1 issue of Optics Letters (DOI:10.1364/OL.33.001545; p. 1545), the researchers described the design and testing of a novel instrument that can capture high-resolution light scattering patterns for sizes well below the resolution of conventional cloud imaging probes. The instrument is composed of a temperaturecontrolled light scattering chamber with a tapered aerosol inlet tube and a vent, an intensified CCD camera, a particle detection trigger photomultiplier detector, a pellicle beam splitter, and a frequencydoubled NdYAG laser. Air flows through the inlet tube at $80 \mathrm{~m} / \mathrm{s}$ and exits into the scattering chamber with a flow diameter of $2.5 \mathrm{~mm}$, where it crosses the laser beam. The pattern of light scattered by individual particles carried in the flow is then captured by the optical system, with a small percentage of light being directed by the pellicle beam splitter onto the detector and the remainder directed to the ICCD camera. Particle size is determined by the magnitude of the signal produced by the photomultiplier detector, and the same signal is used to trigger the ICCD camera to record a high-resolution image of the particle's scattering pattern. This was demonstrated in preliminary experiments, using the University of Manchester Icing Cloud Chamber where ice crystals ranging from $3.5 \mu \mathrm{m}$ to $22 \mu \mathrm{m}$ in diameter were observed for a range of temperatures and humidity. The ice crystals displayed scattering typical of the hexagonal symmetry found in ice. Interpretation of the scattering patterns is required to precisely determine the shape of the crystals. Currently, for less complex crystal shapes, understanding of crystal morphology is achieved by inversion of scattering patterns using theoretical scattering models or by comparison with scattering patterns from known crystal shapes. Future progress in modeling will allow for increased accuracy of the interpretation of scattering patterns for complex crystal shapes. The research in this study was able to uncover information on the morphology and size
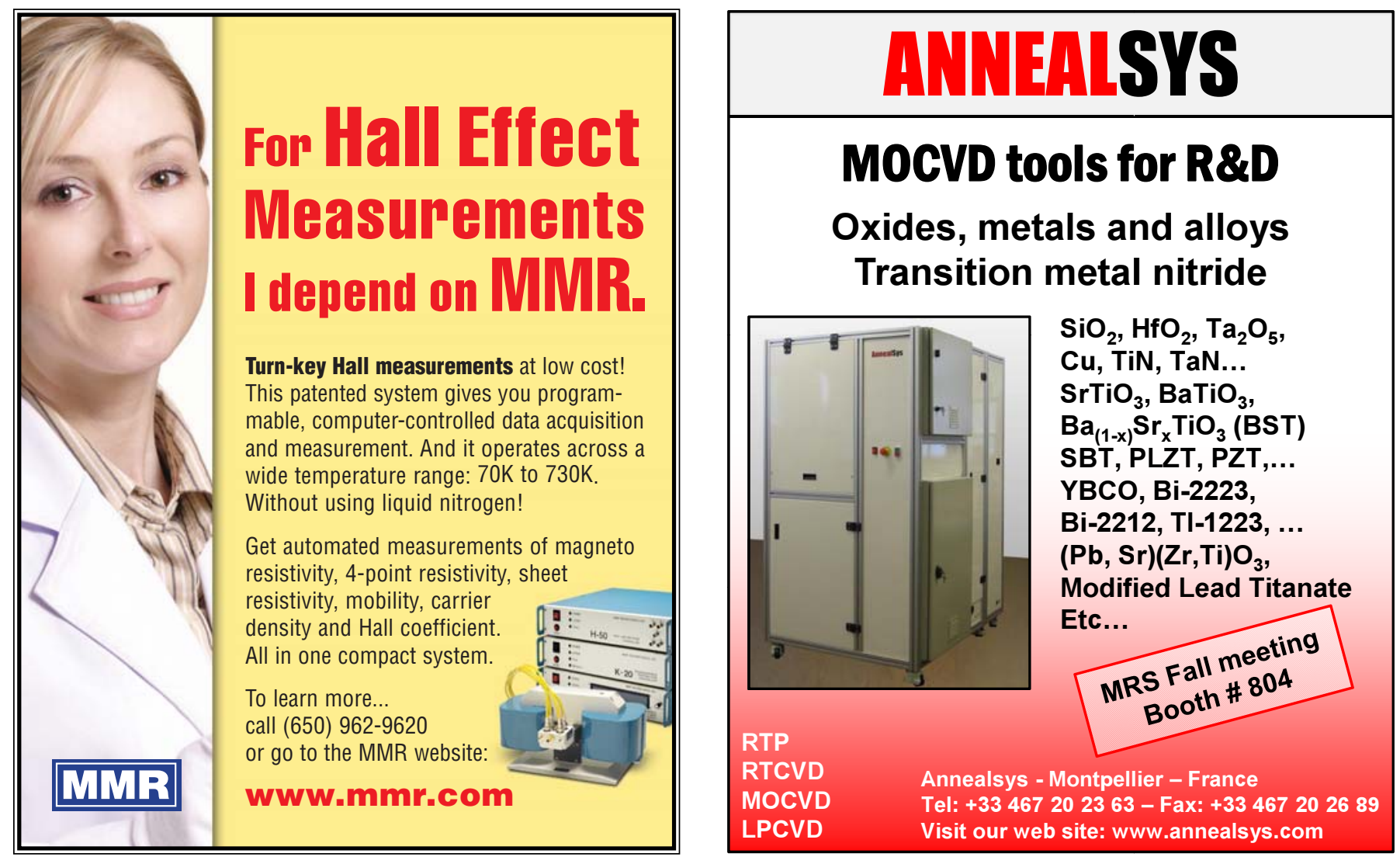
of ice crystals that had previously been beyond the ability of current ice crystal scattering instruments to characterize. The researchers said that this instrument is designed to be used for laboratory studies, as cited here, and also can be mounted on aircraft with specially designed fuselage ports for studies of ambient air.

TARA D. WASHINGTON

\section{Colloidal Single-Sized \\ Nanocrystal Ensemble Exhibits Bright, Narrow-Band PL}

Nanocrystals and nanoparticles are currently of great interest for a variety of applications, ranging from precision polishing of surfaces to bio-oriented, energyassociated, environmental-related, and security-allied applications. In particular, colloidal semiconductor quantum dots, which are ultra-small nanocrystals and spherical in shape, have demonstrated excellent optical properties such as narrow photoemission and broad absorption, and thus create potential for new applications as well as to replace traditional fluorescence dye molecules in existing areas. Most processes for synthesis of colloidal quantum dots yield a dispersion of particle sizes, which results in photoemission linewidths (e. g., 30 nm) that are substantially broader (due to inhomogeneous broadening) than the linewidth of a single quantum dot. K. Yu and colleagues at the National Research Council of Canada have now synthesized single-sized nanocrystal ensembles exhibiting bright bandgap photoluminescence (PL) but with bandwidth as narrow as that from a single quantum dot (e.g., $\sim 10 \mathrm{~nm}$ ).

Yu's group has developed a methodology of molecular synthesis for the precise formation of colloidal semiconductor nanocrystals using a synthetic approach

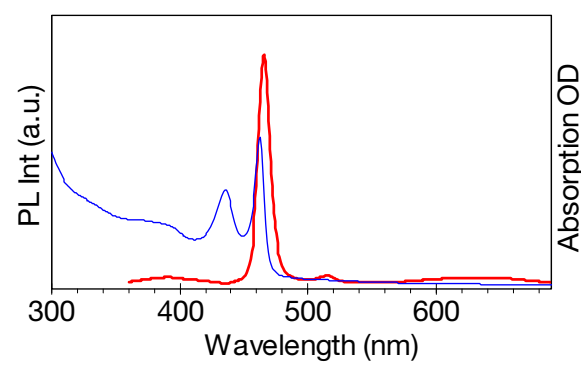

Figure 1. CdSe magic-sized nanocrystal Family 463 dispersed in toluene demonstrates pure-color light absorption (blue line, right y axis) and emission (red line, left y axis). Reprinted with permission from the Journal of Physical Chemistry C 112(36) (2008) p. 13805. (C2008 American Chemical Society. analogous to bottom-up self-assembly. This research group specializes in the development of colloidal single-sized nanocrystal ensembles, also called magicsized nanocrystal (MSN) ensembles, using semiconductor quantum dots such as cadmium selenide (CdSe). The MSN ensembles have the special optical property of sharp light absorption near the absorption band edge and pure-color emission due to the absence of inhomogeneous broadening rising from their single-size property, with an optical response equivalent to one single quantum dot. The group's latest nanotechnology results on molecular synthesis were reported in the September 11 issue of the Journal of Physical Chemistry C (DOI: 10.1021/ jp803845n; p. 13805).

The molecular synthesis of single-sized ensembles simplifies handling and promises to be practical in large-scale production with high reproducibility. This ready approach uses a fatty acid as surface ligands, with 1-octadecene as the reaction medium. For CdSe, cadmium acetate dihydrate and elemental selenium are the sources of $\mathrm{Cd}$ and Se, respectively. With low acid-to-Cd and high Cd-to-Se feed molar ratios, all of these chemicals are loaded at room temperature in a reaction flask, and the growth of the CdSe MSNs is carried out at $120^{\circ} \mathrm{C}-240^{\circ} \mathrm{C}$. This synthetic approach allows long periods of growth and annealing at elevated temperatures, resulting in high-quality CdSe MSNs showing strong bandgap PL.

Three different ensembles were reported, classified as Family 513, Family 463, and Family 395, based on their lowest energy absorption peaks in nanometers. Each of the three MSN ensembles reported has a narrow bandwidth. For example, Family 463 emits near $465 \mathrm{~nm}(2.67 \mathrm{eV})$ (shown in Figure 1), and its bandgap nonresonance photoluminescence bandwidth at room temperature is only $7.6 \mathrm{~nm}$. Such a narrow nonresonant bandwidth has been observed in the past only in the emission spectrum of a single dot rather than an ensemble.

Yu's team has carefully evaluated the growth kinetics of these magic-sized nanocrystals, trying to identify a previously unknown reaction growth mechanism. Their experimental data demonstrate the possibility of optimizing reactions to produce desired nanocrystals and to translate laboratory experiments into product applications. Presently, the researchers are extending their efforts beyond CdSe to the molecular synthesis of various other compositions with bandgap photoluminescence and to the exploration of the formation mechanism.

GOPAL RAO
ANGSTROM
SCIENCES
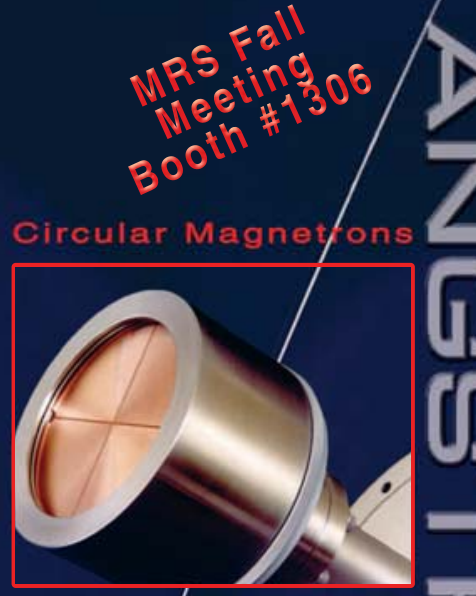

(1)

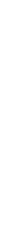

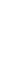
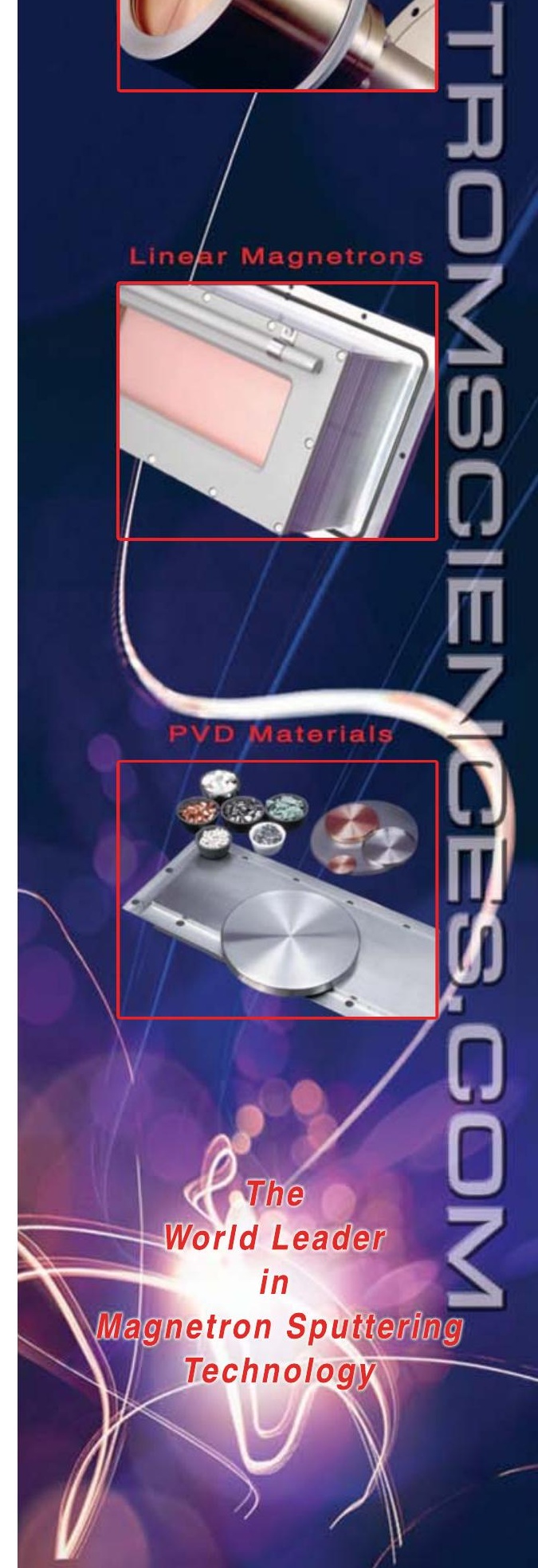